Carina Rossa*

ORCID: 0000-0001-7290-0217

Città del Vaticano

\title{
Università e scuola. Verso il rilancio delle università in uscita. IV Congresso Internazionale delle Cattedre Scholas, Castelgandolfo, 27-29 giugno 2018
}

Il pensiero di Papa Francesco, di cui abbiamo testimonianza in quanto formulato in diversi incontri, discorsi, documenti e pubblicazioni, è particolarmente abbondante e ricco di riflessioni riguardo alla pedagogia e l'approccio sociale, tanto che il Papa viene considerato un grande Maestro del nostro tempo. La sua autorità è riconosciuta ben oltre i confini della Chiesa cattolica e di coloro che si dichiarano cristiani. Questo pensiero non solo merita di essere conosciuto e reso noto, ma è necessario che sia studiato con attenzione e ascoltato meticolosamente. Il messaggio che Papa Francesco esprime si rivolge alle donne e agli uomini di oggi, e offre, in un mondo lacerato da guerre, ingiustizie, incomprensioni e intolleranze, la prospettiva di costruzione di un nuovo umanesimo di cui tutti abbiamo bisogno.

Per approfondire il pensiero di Papa Francesco riguardo all'educazione ed estrarne le linee guida dell'agire pedagogico nei diversi contesti in cui

* Carina Rossa PhD, laureata in Scienze Psicopedagogiche alla Pontificia Universidad Catolica Argentina, Dottore in Pedagogia presso la Libera Università Maria Santissima Assunta (LUMSA) a Roma; Membro del Consiglio Direttivo e Coordinatrice di Area della Fondazione Pontificia Scholas Occurrentes presso la Santa Sede. Indirizzo: Piazza San Calisto 16, 00120, Città del Vaticano, e-mail: carina.rossa@scholasoccurrentes.org 
vengono educati i giovani, è nata l'idea delle Cattedre Scholas, uno spazio accademico qualificato che si propone come obiettivo proprio lo studio, l'approfondimento, la realizzazione e la promozione della pedagogia di Papa Francesco. Esse sono diffuse in tutto il mondo ma si ritrovano ogni anno in un appuntamento molto preciso per condividere le loro ricerche: il Congresso Internazionale. Il primo si è svolto nella prestigiosa Pontificia Accademia delle Scienze in Vaticano; il secondo a Valencia, Spagna; il terzo a Gerusalemme nella Hebrew University.

Il IV Congresso Internazionale delle Cattedre Scholas ha avuto sede nella Villa Pontificia di Castel Gandolfo dal 27 al 29 giugno 2018 ed è stato dedicato alla riflessione sul ruolo degli istituti di istruzione di grado superiore, anche essi "in uscita" di fronte ai grandi problemi dell'umanità. Il Congresso ha coinvolto 245 partecipanti, provenienti da 30 paesi, e rappresentanti di 75 Università dei 5 continenti. Tra esse possiamo citare: 1'Università Alma Mater di Bologna (Italia), la Fordham University (Stati Uniti); l'Ishik University, università musulmana (Iraq); 1'Università Cattolica del Congo; l'Unilasalle Canoas, al centro del Brasile.

I tre assi tematici principali sono stati: Educare all'Umanesimo Solidale; Sfide dello sviluppo integrale alla luce della Laudato Sii; Dialogo interreligioso e multiculturale nella costruzione della Pace. I numerosi e prestigiosi relatori hanno affrontato i temi da diverse prospettive disciplinari e culturali. Tra i principali esponenti di Educare all'Umanesimo Solidale citiamo S.E. Mons. Vincenzo Zani (Città del Vaticano), Segretario della Congregazione per l'Educazione Cattolica della Santa Sede; il Prof. Andrew Furco (Stati Uniti) della Minnesota University, noto come uno dei più esperti ricercatori del Service Learning a livello mondiale e Fr. Michael Smith SJ (Nuova Zelanda) dell'International Education Officer for Jesuit Refugee Service, soltanto per citarne alcuni. Sulle Sfide dello sviluppo integrale alla luce della Laudato Sii, il Prof. Francisco Carballo (UK) del Dipartimento di Politica e Relazioni Internazionali di Goldsmiths, Università di Londra; Augusto Zampini (Argentina), teologo del Dicastero dello Sviluppo Umano Integrale della Santa Sede; sul tema Dialogo interreligioso e multiculturale nella costruzione della Pace, il Prof. Alfonso de Toro (Germania), Direttore del Seminario di Ricerca Ibero-americana e del Centro di Ricerca Francofono dell'Università di Leipzig, Germania; il Rabbino Daniel Goldman (Argentina) dell'Istituto di Dialogo Interreligioso; il Dr. Flavio Lotti (Italia) del Coordinamento Nazionale enti locali per la Pace e diritti umani. 


\section{Le Cattedre Scholas}

Le Cattedre si propongono come luoghi di riflessione e di azione, collegati in una rete, in cui ogni soggetto (docenti, ricercatori, studenti) si arricchisce nell'incontro con l'altro, attraverso i vincoli che si sviluppano tra le varie università, le scuole e i progetti della piattaforma scholas.social. Nelle Cattedre Scholas il dialogo tra le diverse religioni assume un carattere particolare. La composizione multiculturale e multireligiosa che oggi caratterizza le diverse università aderenti al progetto Scholas, rappresenta una grande risorsa in quanto fonte di arricchimento e stimolo per la costruzione di un mondo più solidale e pacifico. "Il dialogo è molto importante per la propria maturità, perché nel confronto con l'altra persona, nel confronto con le altre culture, anche nel confronto sano con le altre religioni, uno cresce: cresce, matura [...] Questo dialogo è quello che fa la pace", ha detto Papa Francesco ${ }^{1}$.

Fedeli al metodo dell'incontro e all'attenzione alla realtà, le Cattedre Scholas prendono come punto di partenza i problemi e le questioni attuali che le parole di Papa Francesco incessantemente richiamano alla nostra attenzione. Tali problemi e questioni hanno bisogno di essere approfonditi non solo con considerazioni di tipo meramente accademico, ma anche attraverso metodi di educazione attiva, facendo coincidere la conoscenza con l'azione, la discussione e l'analisi con l'intervento concreto di trasformazione della realtà.

\section{Università "in uscita"}

Papa Francesco quest'anno ha voluto emanare una nuova Costituzione Apostolica che riguarda le Università e Facoltà ecclesiastiche; si tratta della Costituzione Veritatis Gaudium ${ }^{2}$ che fa parte del suo programma di rinnovamento. In esso si legge fra l'altro:

${ }^{1}$ Francesco, Discorso agli studenti e ai professori del Collegio Seibu Gakuen Bunry Junior High School di Saitama, Tokyo. 21 agosto 2013 (Vatican: Libreria Editrice Vaticana): http://w2. vatican.va/content/francesco/it/speeches/2013/august/documents/papa-francesco_20130821_ collegio-saitama-giappone.html [9.10.2018].

${ }^{2}$ Francesco, Veritatis gaudium (Vatican: Libreria Editrice Vaticana, 2017). 
È giunto ora il momento in cui questo ricco patrimonio di approfondimenti e di indirizzi, verificato e arricchito per così dire "sul campo" dal perseverante impegno di mediazione culturale e sociale del Vangelo messo in atto dal Popolo di Dio nei diversi ambiti continentali e in dialogo con le diverse culture, confluisca nell'imprimere agli studi ecclesiastici quel rinnovamento sapiente e coraggioso che è richiesto dalla trasformazione missionaria di una Chiesa "in uscita"”.

Il nuovo rilancio delle Università è fondato su quattro criteri:

a) "La mistica del noi": lievito della fraternità universale che cerca di dare concretezza alla dimensione sociale dell'evangelizzazione, dando priorità agli ultimi, allo scarto della società: "che sa guardare alla grandezza sacra del prossimo, che sa scoprire Dio in ogni essere umano, che sa sopportare le molestie del vivere insieme aggrappandosi all'amore di Dio, che sa aprire il cuore all'amore divino per cercare la felicità degli altri come la cerca il loro Padre buono"4.

b) "Il dialogo a tutto campo": come esigenza intrinseca per fare esperienza comunitaria e come pratica concreta della Cultura dell'Incontro. Esso "invita a favorire il dialogo con i cristiani appartenenti alle altre Chiese e comunità ecclesiali e con coloro che aderiscono ad altre convinzioni religiose o umanistiche", e insieme a tenersi "in relazione con gli studiosi delle altre discipline, siano essi credenti o non credenti", cercando "di ben intendere e valutare le loro affermazioni, e di giudicarle alla luce della verità rivelata"s. Da ciò deriva un ripensare i curricula a diversi livelli: disciplinare, interdisciplinare, pedagogico e didattico.

c) "L'inter e la trans-disciplinarietà" esercitate con sapienza e creatività. Si tratta di offrire, attraverso i diversi percorsi proposti dagli studi, una pluralità di saperi, corrispondente alla ricchezza multiforme del reale nella luce dischiusa dall'evento della Rivelazione, che sia al tempo stesso armonicamente e dinamicamente raccolta nell'unità della sua sorgente trascendente e della sua intenzionalità

3 Ibidem, no. 3.

4 Ibidem, no. 92 .

5 Ibidem, no. 4b; cf. Concilio Ecumenico Vaticanum II, Gaudium et spes (Vatican: Libreria Editrice Vaticana), no 62, http://www.vatican.va/archive/hist_councils/ii_vatican_council/ documents/vat-ii_const_19651207_gaudium-et-spes_it.html [9.10.2018]. 
storica e metastorica, quale è dispiegata escatologicamente in Cristo Gesù 6 .

d) "Fare 'rete' tra le diverse istituzioni" che, in ogni parte del mondo, coltivano e promuovono gli studi ecclesiastici, attivando con decisione le opportune sinergie anche con le istituzioni accademiche dei diversi Paesi e con quelle che si ispirano alle diverse tradizioni culturali e religiose, dando vita al contempo a centri specializzati di ricerca finalizzati a studiare i problemi di portata epocale che investono oggi l'umanità, giungendo a proporre opportune e realistiche piste di soluzione.

In questo senso si sollecita una ricerca fatta a contatto con la realtà, orientata alla cura della Casa Comune, a difesa dei poveri, nella costruzione di reti di rispetto e fraternità.

\section{Quali sfide per le Cattedre Scholas 'in uscita'?}

Infine la conclusione: al confronto ed alla luce della Costituzione Apostolica Veritatis Gaudium si sono aperte nuove sfide, che descriviamo brevemente qui di seguito.

a) Sfide accademiche: Si percepisce il bisogno di promuovere metodi di insegnamento "oltre l'aula", nel quale gli studenti possano entrare in contatto con la comunità ed il territorio come fonte di insegnamento significativo e pratiche applicate alla soluzione di problemi reali. Con creatività si dovrebbero trovare e immaginare strategie perché lo studente possa imparare ad apprendere, utilizzando il linguaggio "della testa, della mano e del cuore", in funzione di conoscenze socialmente utili. Occorre una articolazione maggiore tra le discipline, la docenza, la ricerca e la responsabilità sociale universitaria. In questo senso tra le metodologie più frequentemente usate nelle Cattedre Scholas che rispondono a questo criterio troviamo la proposta pedagogica dell'Apprendimento Servizio.

b) Ricerca e gestione del sapere: La ricerca dovrebbe essere indirizzata allo sviluppo di saperi socialmente utili e generati dalla comunità in rete con altre università. Si dovrebbero generare spazi di Re-

${ }^{6}$ Francesco, Veritatis, no. 4 c.

7 Discorso del Santo Padre Francesco in occasione della Chiusura del IV Congresso Mondiale Educativo delle "Scholas Occurrentes". Aula del Sinodo, 5 febbraio 2015. 
search Action in grado di rispondere in modo significativo e critico nei confronti dell'imperante sistema economico caratterizzato dalla "cultura dello scarto", collegando educazione ed economia al servizio dei popoli. Inoltre, ricercatori e docenti devono lavorare sulla stessa problematica nello stesso posto creando sinergia tra i saperi in modo interdisciplinare. In questa linea le Cattedre Scholas hanno costituito durante l'attuale anno accademico 12 gruppi di lavoro e agiscono in rete tra cinque o sei università che hanno scelto lo stesso tema. I temi di ricerca sono: Sport e educazione; Arte e educazione; Educazione e nuove tecnologie; Educazione inclusiva; Cultori dell'Incontro; Mobilità internazionale; Movimenti sociali; Mobilità umana e rifugiati; Educazione e sviluppo sostenibile; Pace sostenibile; Dialogo multiculturale e interreligioso; Responsabilità sociale dell'Università; Patto educativo.

c) Impegno sociale o Terza missione: Implementare progetti di sviluppo (soggetti) collegati alla ricerca applicata, fonte di risorse didattiche per la comunità universitaria. Si prevede inoltre un aumento significativo del volontariato degli studenti attraverso il quale possano arrivare ad un apprendimento più significativo. Per raggiungere questo obiettivo Scholas offre due programmi: Scholas.social, piattaforma di progetti e buone prassi educative, online e offline, fonte per la ricerca, ed il programma Scholas.Cittadinanza attraverso il quale studenti di scuole medie di una stessa città si incontrano e lavorano insieme per i problemi della comunità.

d) Gestione interna dell'Università: L'Università va vista come una comunità socialmente esemplare che mette in pratica i cinque criteri della Veritatis Gaudium. Si spera che essa possa essere anche un modello di sviluppo sostenibile e cioè che, come istituzione, promuova politiche di protezione dell'ambiente e di sviluppo sociale.

Come ogni anno l'appuntamento del Congresso delle Cattedre Scholas è un'opportunità di aggiornamento, di messa a punto dei criteri di qualità, di apertura a nuove sfide ma soprattutto d'incontro tra accademici amici che vivono la "Mistica del Noi". Il 2019 ci attende con nuove sfide in una nuova sede che ci accoglie: la Fordham University a New York.

${ }^{8}$ Francesco, Udienza Generale. Piazza San Pietro, 5 giugno 2013 (Vatican: Libreria Editrice Vaticana): http://w2.vatican.va/content/francesco/it/audiences/2013/documents/papa-francesco_20130605_udienza-generale.html [9.10.2018]. 